\title{
The impact of an inpatient electronic prescribing system on prescribing error causation: a qualitative evaluation in an English hospital
}

\begin{abstract}
Introduction

Few studies have applied a systems approach to understanding the causes of specific prescribing errors in the context of hospital electronic prescribing (EP). A comprehensive understanding of underlying causes is essential for developing effective interventions to improve prescribing safety. Our objectives were to explore prescribers' perspectives of the causes of errors occurring with EP and to make recommendations to maximise benefits and minimise risks.
\end{abstract}

\section{Methods}

We studied a large hospital using inpatient EP. From April to June 2016, semi-structured interviews were conducted with purposively sampled prescribers involved with a prescribing error. Interviews explored prescribers' perceived causes of the error and views about EP, and were audio-recorded and transcribed verbatim. Data were thematically analysed against a framework based on Reason's accident causation model, with a focus on identifying latent conditions.

\section{Results}

Twenty-five interviews explored causes of thirty-two errors. Slips and rule-based mistakes were the most common active failures. Error causation was multifactorial; environmental, individual, team, task and technology error-producing conditions were all influenced by EP. There were three broad groups of latent conditions: the EP system's functionality and design; the organisation's decisions around EP implementation and use; and prescribing behaviours in the context of EP.

\section{Conclusions}

Errors were associated with the design of EP itself and its integration within the healthcare environment. Findings suggest that EP vendors should focus on revolutionising interface design and usability issues, bearing in mind the wider healthcare context in which such software is used. Healthcare organisations should draw upon human factors principles when implementing EP. Consideration of work environment, infrastructure, training, prescribing responsibilities and behaviours should be considered to address local issues identified. 


\section{INTRODUCTION}

Prescribing errors are a common source of preventable harm in hospitals worldwide. ${ }^{1}$ In the UK, prescribing errors occur in $7.5-14.7 \%$ of inpatient medication orders, harming an estimated $1-2 \% .^{2-6}$ Electronic prescribing (EP) is a potentially important intervention to reduce errors $^{7-10}$ and its use strongly advocated. ${ }^{11,12}$ EP provides many potential benefits over handwritten medication orders, including completeness, standardisation, legibility, audit trails, and decision support.-9,13 However, prescribing errors are not mitigated entirely; EP may contribute to new types of error not seen with paper. ${ }^{14-16}$

Prescribing is a complex process involving consideration of the patient's clinical condition, medical history, evidence base, medication availability, potential adverse effects and patient preference, followed by correct generation of the medication order. ${ }^{17}$ This multi-faceted process makes prescribing a common source of error. ${ }^{17,18}$ The application of human factors principles and a systems approach has become well established within healthcare;19,20,21 such concepts acknowledge the "complex interplay between people, tasks, technologies, organisations and environments". ${ }^{22}$ In particular, Reason's model of accident causation (appendix 1$)^{23-25}$ has been used to explore the causes of prescribing errors. ${ }^{2,18,26-29}$ This model assumes that 'latent conditions' create an environment where 'error-producing conditions' are more likely to occur and thus cause 'active failures' at the front-line.23,27 Latent conditions are the management decisions, organisational processes and cultural issues that create risks within the system; error-producing conditions are specific factors associated with the patient, individual, team, task, technology or the environment that directly affect front-line operations. Since latent conditions are the precursor to error-producing conditions, it is important to identify and understand these to develop a proactive risk management strategy and develop effective interventions. ${ }^{23,30}$

There is a wealth of information about causes of prescribing errors associated with paperbased prescribing. 2,18,26-29 Such studies have sourced perceptions of error causation from prescribers themselves, as it is argued that cause is "inextricably linked with knowing the intention of the person" involved. ${ }^{27}$ These studies suggest that causes are multifaceted and influenced by various error-producing and latent conditions. . $^{218,26-29}$ Other studies suggest that EP can give rise to new types of error, attributed to technology design, functionality and usability issues. ${ }^{14-16,31} \mathrm{~A}$ socio-technical approach has identified workflow and communication issues as contributory factors. ${ }^{14,15,32-34}$ A recent review provides valuable insights into prescribing error causation, focussing on issues associated with the EP system itself and user-EP interaction. ${ }^{31}$ However, there has been little work exploring causes of prescribing errors using models of human error in the context of EP. Such analysis would facilitate identification of latent conditions that influence errors in practice, leading to recommendations for targeted interventions. 
Our objectives were to describe the causes of prescribing errors associated with EP from prescribers' perspectives using Reason's accident causation model, explore the associated latent conditions, and make recommendations to maximise benefits and minimise risks associated with inpatient EP.

\section{METHODS}

\section{Design and setting}

We used an established qualitative approach to exploring prescribing error causality. ${ }^{18,26-28}$

We studied all twenty-four medical and surgical wards in a 500 bed English teaching hospital that implemented a commercially available inpatient EP system in 2015. Six critical care and paediatric wards were excluded as they did not use EP. Prior to EP, prescribers hand-wrote medication orders on paper drug-charts. Using EP, prescribers accessed patient records using an access card and secure log-in on a desktop computer or computer-on-wheels (COW). Medication orders were generated from drop-down lists to select the required drug, dose, route and administration times. The available decision support comprised dosing regimen suggestions ("order-sentences") for commonly used drugs, lists of multiple orders for particular situations ("order-sets"), patient-specific drug-allergy notifications, and reminder alerts for the timely assessment of thromboprophylaxis risk and medication review.

\section{Participant recruitment}

All pharmacists working on included wards were requested to report prescribing errors identified as part of their normal clinical duties to a research pharmacist; this did not replace the need to report through the organisation's incident reporting system. The researcher electronically reviewed the medication order to identify the prescriber(s) involved and determine whether it met our definition a prescribing error (appendix 2). ${ }^{35}$ Prescribers who had generated incorrect medication orders were purposively sampled ${ }^{36}$ to maximise variation in error types, prescriber grades and clinical specialties. We categorised types of prescribing error based on a published taxonomy ${ }^{37}$; categories specific to EP were iteratively incorporated. Prescribers were contacted in person or by telephone within 96 hours of the error to aid recall, ${ }^{18}$ briefed about study objectives and invited to participate; alternatively an email sent inviting them to contact the researcher for further information.

\section{Data collection}

Face-to-face semi-structured interviews were conducted as soon as possible in hospital locations chosen by participants. Interviews were guided by an interview schedule (appendix 3) developed from existing literature ${ }^{2,3,18,24,38}$ and piloted with one doctor beforehand. Questions for all participants focussed on the circumstances and causes surrounding the error and their experiences of the EP system; probes and clarification questions were added as necessary. Interviews were audio-recorded and transcribed verbatim by a commercial 
transcriber; one was transcribed by the researcher because confidential information was potentially audible in the background. Both researchers were pharmacists employed by the hospital organisation with clinical, medication safety, and qualitative research experience. The researcher conducting interviews was unknown to participants prior to the study.

\section{Data analysis}

Data were analysed thematically using NVivo software. Data relating to error causation were analysed deductively using a framework based on Reason's accident causation model. ${ }^{23-25}$ Data were mapped against the model's categories of active failure (slip, lapse, rule-based mistake, knowledge-based mistake or violation) and error-producing conditions (patient, individual, team, environmental, task or technology factors); analysis and interpretation focussed on linking these to their underlying latent conditions (organisational culture, management strategy or external influences). We also considered whether error-producing conditions were specific to EP or applicable to both paper and EP. Interviews continued until the researcher judged that theoretical saturation had been reached. ${ }^{39}$ Coding of three interviews was checked by a second researcher; no disagreements were identified. In a separate process, both researchers categorised each active failure and any disagreements were resolved via discussion.

\section{Ethical considerations}

The study was approved locally; NHS ethics approval was not required. Lead clinicians were encouraged to inform members of their teams about the study. Participants provided written informed consent prior to interview.

\section{RESULTS}

\section{Participants}

Pharmacists reported 193 prescribing errors by 122 prescribers during April to June 2016; 81 prescribers were invited to participate (appendix 4). Twenty-five interviews exploring 32 errors were conducted. Participants comprised one nurse prescriber and 24 doctors: seven of foundation year 1 (FY1); three foundation year 2 (FY2); eight senior house officers (SHOs); four registrars and two consultants. ${ }^{40}$ Participants represented medical $(n=11)$ and surgical $(n=13)$ specialities. Interviews lasted from 8 to 35 minutes

\section{Types of prescribing errors and active failures}

There were 11 types of error explored during interviews (appendix 5); a primary active failure was identified for each error (table 1). 
Table 1. Examples of the types of prescribing error explored during interviews and their active failure classification. Appendix 6 presents details of all errors explored during interviews.

\begin{tabular}{|c|c|c|}
\hline \multirow{2}{*}{$\begin{array}{l}\text { Type of active } \\
\text { failure }(n ; \text { of a } \\
\text { total of } 32)\end{array}$} & \multicolumn{2}{|c|}{ Examples of prescribing errors explored during interviews } \\
\hline & $\begin{array}{l}\text { Type of } \\
\text { prescribing error }\end{array}$ & Brief description of prescribing error \\
\hline \multirow{2}{*}{ Slip (4) } & $\begin{array}{l}\text { Incorrect dosage } \\
\text { units }\end{array}$ & 150 tablets of irbesartan selected instead of $150 \mathrm{mg}$. \\
\hline & Incorrect drug & $\begin{array}{l}\text { Ceftriaxone selected instead of cefuroxime for gastro- } \\
\text { intestinal surgery prophylaxis. }\end{array}$ \\
\hline Lapse (1) & $\begin{array}{l}\text { Drug prescribed } \\
\text { is not indicated } \\
\text { for patient }\end{array}$ & $\begin{array}{l}\text { Doxycycline was intended to be stopped but was } \\
\text { unintentionally continued. }\end{array}$ \\
\hline $\begin{array}{l}\text { Knowledge-based } \\
\text { mistake (1) }\end{array}$ & Incorrect dose & $\begin{array}{l}\text { Pregabalin initiated at } 75 \mathrm{mg} \text { daily, however a reduced dose } \\
\text { of } 25 \mathrm{mg} \text { daily was required due to severe renal impairment. } \\
\text { The prescriber was aware they lacked knowledge to } \\
\text { appropriately reduce the dose so consulted senior advice } \\
\text { when prescribing. }\end{array}$ \\
\hline $\begin{array}{l}\text { Rule-based } \\
\text { mistake: } \\
\text { Failure to apply a } \\
\text { good rule (5) }\end{array}$ & Incorrect dose & $\begin{array}{l}\text { Co-amoxiclav [amoxicillin and clavulanic acid] prescribed at } \\
1.2 \mathrm{~g} \text { three times a day however a reduced dose of twice a } \\
\text { day was required due to renal impairment. The prescriber } \\
\text { knew a dose reduction is required in renal impairment } \\
\text { although failed to consider renal function when prescribing. }\end{array}$ \\
\hline \multirow{3}{*}{$\begin{array}{l}\text { Rule-based } \\
\text { mistake: } \\
\text { Misapplication of } \\
\text { a good rule (5) }\end{array}$} & $\begin{array}{l}\text { Drug prescribed } \\
\text { is not indicated } \\
\text { for patient }\end{array}$ & $\begin{array}{l}\text { Trimethoprim prescribed for treatment of urinary tract } \\
\text { infection, however was not suitable for the patient as their } \\
\text { midstream urine sensitivity suggested resistance to } \\
\text { trimethoprim. }\end{array}$ \\
\hline & $\begin{array}{l}\text { Failure to take } \\
\text { into account drug } \\
\text { interaction }\end{array}$ & $\begin{array}{l}\text { Ciprofloxacin prescribed for urinary tract infection without } \\
\text { considering if there was an interaction with duloxetine which } \\
\text { was already prescribed. }\end{array}$ \\
\hline & $\begin{array}{l}\text { Drug prescribed } \\
\text { is contra- } \\
\text { indicated }\end{array}$ & $\begin{array}{l}\text { The adverse effect of tramadol lowering seizure threshold } \\
\text { was not considered and prescribed for a patient with a brain } \\
\text { injury and alcohol induced seizures. }\end{array}$ \\
\hline \multirow{5}{*}{$\begin{array}{l}\text { Rule-based } \\
\text { mistake: } \\
\text { Application of an } \\
\text { inappropriate rule } \\
\text { (14) }\end{array}$} & $\begin{array}{l}\text { Duplicated } \\
\text { therapy }\end{array}$ & $\begin{array}{l}\text { Insulin (NovoMix30®) prescribed although was not required } \\
\text { as the patient was already receiving insulin by variable rate } \\
\text { intravenous infusion. }\end{array}$ \\
\hline & $\begin{array}{l}\text { Duplicated } \\
\text { therapy }\end{array}$ & $\begin{array}{l}\text { The medication order for oral co-amoxiclav [amoxicillin and } \\
\text { clavulanic acid] } 625 \mathrm{mg} \text { three times a day was duplicated }\end{array}$ \\
\hline & $\begin{array}{l}\text { Medication } \\
\text { omitted when } \\
\text { clinically indicated }\end{array}$ & $\begin{array}{l}\text { Abacavir was unintentionally not prescribed on admission to } \\
\text { hospital because the prescriber was unaware that it was the } \\
\text { patient's usual medication. }\end{array}$ \\
\hline & $\begin{array}{l}\text { Prescribing on an } \\
\text { invalid } \\
\text { prescription }\end{array}$ & $\begin{array}{l}\text { All medications were ordered in an incorrect patient } \\
\text { encounter referring to a patient's previous admission, so the } \\
\text { prescription was invalid and inactive. }\end{array}$ \\
\hline & $\begin{array}{l}\text { Incorrect dosing } \\
\text { schedule }\end{array}$ & $\begin{array}{l}\text { Meropenem was switched to ertapenem, however the first } \\
\text { dose of ertapenem was scheduled for the following day } \\
\text { however was intended to be prescribed immediately. }\end{array}$ \\
\hline $\begin{array}{l}\text { Violation } \\
\quad(2)\end{array}$ & Incorrect route & $\begin{array}{l}\text { Oral (rather than intravenous) vancomycin was prescribed } \\
\text { for hospital acquired pneumonia. The patient received the } \\
\text { correct route in practice, however the electronic chart used } \\
\text { for record purposes was incorrect and not updated. }\end{array}$ \\
\hline
\end{tabular}




\section{Causes of prescribing errors}

Multiple error-producing conditions were described; their perceived association with EP is presented in table 2. There were three broad groups of latent conditions: 1) EP system functionality and design; 2) organisational decisions around EP implementation and use, and 3) prescribing behaviours in the context of EP; the results relating to each of these are next described in turn, highlighting the influence of latent conditions on subsequent error-producing conditions and active failures. Quotations are illustrative of key themes and findings. 
Table 2: Error-producing conditions reported as contributory factors to prescribing errors

\begin{tabular}{|c|c|c|}
\hline $\begin{array}{l}\text { Category of } \\
\text { error- } \\
\text { producing } \\
\text { conditions }\end{array}$ & $\begin{array}{c}\text { Error-producing conditions applicable } \\
\text { to both electronic and paper- } \\
\text { prescribing }\end{array}$ & $\begin{array}{l}\text { Error-producing conditions } \\
\text { specifically associated with electronic } \\
\text { prescribing (EP) }\end{array}$ \\
\hline $\begin{array}{l}\text { Patient } \\
\text { factors }\end{array}$ & $\begin{array}{l}\text { - } \text { Complex patient } \\
\text { - } \quad \text { Patient perceived to be unreliable } \\
\text { information source }\end{array}$ & \\
\hline $\begin{array}{l}\text { Individual } \\
\text { factors }\end{array}$ & $\begin{array}{l}\text { - Lack of specific drug knowledge } \\
\text { - Unaware of lack of knowledge } \\
\text { - Unaware of own errors } \\
\text { - Inappropriate self-check process } \\
\text { - Impaired physical or mental well- } \\
\text { being }\end{array}$ & $\begin{array}{l}\text { - Lack of skills using EP system } \\
\text { - Perception that prescribing on EP is } \\
\text { simple and low-risk } \\
\text { - Perception that electronic } \\
\text { documentation is less important than } \\
\text { clinical appropriateness } \\
\text { - Variable training using EP system }\end{array}$ \\
\hline $\begin{array}{l}\text { Team } \\
\text { factors }\end{array}$ & $\begin{array}{l}\text { - Junior staff have main prescribing } \\
\text { role } \\
\text { - Prescribers receive incorrect or } \\
\text { incomplete advice } \\
\text { - Lack of supervision and support } \\
\text { - Expanding prescribing roles to non- } \\
\text { medical staff can result in deskilled } \\
\text { doctors } \\
\text { - Poor communication within large } \\
\text { teams } \\
\text { - Poor communication between } \\
\text { multiple teams } \\
\text { - Prescribers do not receive feedback } \\
\text { - Obout errors } \\
\text { errors }\end{array}$ & $\begin{array}{l}\text { - Senior doctors have limited practical } \\
\text { knowledge using EP }\end{array}$ \\
\hline $\begin{array}{c}\text { Work } \\
\text { environment }\end{array}$ & $\begin{array}{l}\text { - } \quad \text { High workload and time pressures } \\
\text { - } \quad \text { Busy ward rounds and on-call shifts } \\
\text { - Interruptions and distractions }\end{array}$ & $\begin{array}{l}\text { - Limited availability of portable } \\
\text { computers } \\
\text { - Higher workload for individual } \\
\text { operating the computer } \\
\text { - Portable computers considered too } \\
\text { cumbersome } \\
\text { - Prescribing remotely without } \\
\text { observing patient }\end{array}$ \\
\hline $\begin{array}{l}\text { Task } \\
\text { factors }\end{array}$ & $\begin{array}{l}\text { - } \quad \text { Prescribing has low priority } \\
\text { - } \quad \text { Lack of written resources available } \\
\text { - } \text { Misinterpreting prescribing guidelines } \\
\text { - } \quad \text { Patient information not sought }\end{array}$ & $\begin{array}{l}\text { - Pharmacist advice not sought } \\
\text { - Information is fragmented } \\
\text { - Hybrid paper and electronic systems } \\
\text { - Over-reliance on order-sentences }\end{array}$ \\
\hline $\begin{array}{l}\text { Technology } \\
\text { factors }\end{array}$ & & $\begin{array}{l}\text { - Complex prescribing functions within } \\
\text { - UP } \\
\text { - Unfamiliar terminology } \\
\text { - Inappropriate default settings } \\
\text { - Problems interpreting information } \\
\text { - Lifficulty accessing information } \\
\text { - Ung drop-down lists } \\
\text { - Slow log-in process } \\
\text { - Lack of clinical decision support }\end{array}$ \\
\hline
\end{tabular}




\section{EP system functionality and design}

\section{Prescribing functions and usability}

All participants identified that design, usability and functionality issues can negatively influence safety. While interviewees recognised the system's inherent limitations, they believed that local informatics teams should be able to resolve certain problems.

Some commonly used medications were perceived as disproportionately complex to prescribe using EP; these included medications that require variable dosing such as warfarin, courses of fixed duration, and once-only doses. Furthermore, problems arose when precise terminology was required to order medication; doctors considered the requirement to specify certain dose units or additives to be outside of their remit.

"Doctors are very familiar with prescribing potassium in amounts of millimoles rather than in percentages of a solution [...] [and] really struggle with that. (Interview 22, SHO)

"I probably spent about an hour working out how to prescribe a syringe driver [...] it was asking me about the additives. I was like this is not something you do as a doctor, nurses just know what to add." (Interview 11, FY2)

The EP system listed only approved generic medication names as per hospital policy. This was perceived as unhelpful when searching for medications with a commonly used alternative name, such as "vitamin K" for phytomenadione. Two interviewees described prescribing alternative treatments if the first choice could not be located.

“...I wanted to write up a plain bupivacaine epidural infusion. I couldn't find it [...] I don't want to give the guy some fentanyl in his bupivacaine, but I could only find fentanyl one. So I had to stop his systemic opioids and just put him on the fentanyl." (Interview 4, Consultant)

Ordering incorrect drugs or dosage units was associated with slips using keystrokes or selection errors from drop-down lists, sometimes considered too long or in an unintuitive sequence.

"Why do you have to scroll through 100 words beginning with 'l' when IV [intravenous] surely is more common than intrathecal" (Interview 14, SHO)

\section{Interface design}

Participants felt that medication-related interfaces were inadequately designed and difficult to interpret. The default view for ordering medications caused confusion as medications appeared among orders for blood or scan requests, in what was perceived to be a nonsensical order. Additionally, it was felt that essential information was hidden or fragmented due to overcrowded screens or multiple tabs that were inaccessible simultaneously. 
"...it's basically just too much writing on all of the screens, so then you generally pay attention to nothing as opposed to actually seeing anything useful." (Interview 20, SHO)

"...so within the boxes where the medicines are prescribed, you often have to hover over it with a cursor to see the full prescription, and important parts of the prescription can be ignored as a result." (Interview 12, Consultant)

Interpreting previously administered medications was considered particularly challenging because a limited number of drugs were displayed at a time. Respondents reported missing important information such as dose omissions or when medication had been stopped.

"It's not the easiest interface to use. You have to scroll across, across, across [...] And then if they've a long prescription you have to go all the way up and all the way down. It would be easier to have a zoom out tool." (Interview 24, Registrar)

Consequently, respondents admitted not checking some prescriptions at all, with duplications attributed to failing to check the medication already prescribed.

"... when you had a paper chart you would just open it up and quickly flick through and have a look at the drugs, and I don't think we do that as routinely now that we have an electronic chart [...] I think we're all very used to a very visual drug chart, and [the EP system] doesn't provide that same experience." (Interview 15, FY2)

\section{Organisational decisions around EP implementation and use}

\section{Paper and electronic hybrid environment}

Within certain clinical areas, including haemodialysis and emergency units, paper-prescribing was still used. Errors occurred due to confusion caused by the hybrid environment.

“...for acute trauma admission we have [...] a paper drug chart. And frequently we'll put the first dose of phenytoin, which is a loading dose onto that. And so you have to go and find that to check if they've had the loading dose. So sometimes there is confusion related to that first dose as to whether it's been prescribed on [EP system] or on there." (Interview 1, SHO)

\section{EP system training}

Respondents expressed varying levels of confidence and competence using EP. Errors were associated with inability to fully utilise the system, such as not retrieving weight information, or being unable to amend dose frequencies. While some respondents were aware of their knowledge gaps, others erroneously believed certain functions were unsupported by EP. One explanation may be ineffective training; one doctor reported receiving no training at all.

"....you were either physically available and free to have training or you never got trained, and you showed up on the day and then there were no more [paper] drug charts." (Interview 13, FY1) 
In contrast, a participant who was trained as part of the initial EP pilot received intense wardbased training which was not replicated to the same extent during roll-out, suggesting this may be more effective.

"...I got taught how to know which medications have been given and when a stop date was and all these sorts of things. I think lots of people haven't been taught, so there's still lots of doctors who will look at it and go, "I've got no idea if this drug's been given or not."' (Interview 21, FY1)

\section{Prescribing environment and infrastructure}

On ward-rounds where only one COW was used, a heavy workload fell to one individual; multiple COWs were sometimes used to circumvent this. However, in certain clinical areas respondents reported limited or no available COWs. Slow log-in processes were frequently cited as inadequate to support EP and an additional distraction that could lead to slips and lapses. One doctor admitted prescribing using colleagues' accounts instead of switching the user to overcome this.

"And having to find a computer, log onto [EP system] and prescribe it is a bit of a pain, mostly because Trust [NHS organisation] computers are Trust computers. They can take forever, and the [EP] system can be really temperamental at times which can be a real pain...." (Interview 7, SHO)

Respondents suggested that prescribing away from the patient was required when there were insufficient COWs, sometimes perceived as contributing to errors:

"...I prescribed some penicillin... the nurse who was standing with me said "oh I'll put that up straight away". [...] And I had to walk right to the other end of $A$ and $E$ [accident and emergency] which was where my [EP log-in] card was [...] When I got there I typed in penicillin, it flashed up allergy. At which point I sprinted back down to the patient. She was already having it [...] And in fact she didn't have a penicillin allergy [...] But it was a real lesson in making sure it's actually been prescribed in front [of the patient]." (Interview $14, \mathrm{SHO}$

Even where COWs were available, some respondents preferred not to use them because they were too cumbersome.

"... on the postnatal ward, it's an old ward and there's not much space. There's cots, there's relatives, and it's just cumbersome to take [the COW] round between the beds." (Interview 15, SHO)

One doctor also felt that using the COW created a barrier between them and the patient; this led them to prescribe retrospectively rather than during the consultation, which could lead to tasks being forgotten. Additionally, while prescribing without visiting the ward improved efficiency, some felt it should be discouraged due to the potential for errors.

"...the idea that you can prescribe for somebody without being geographically close to them, i.e. having not laid eyes on them, I think will eventually lead to something going wrong or amiss [...] you're depending on someone else's maybe less experienced assessment and some numbers rather than your own clinical acumen." (Interview 20, SHO) 


\section{Prescribing behaviours in the context of EP Expectations of decision support}

Prescribers had positive views of the available decision support. In particular, allergy alerts, order-sentences and order-sets were considered to improve safety and efficiency. However, over-reliance resulted in rule-based mistakes when order-sentences or order-sets were not tailored for the patient.

"There's an anaesthetic care-set... and up comes all the things we anaesthetists like... And basically I just zip down there, tick, tick, tick, all the things I feel [the patient needs]. [...] I did this again last week actually, and I looked at the chart [and thought] "Oh my goodness this guy's on two paracetamols"." (Interview 4, Consultant)

It was recognised that misplaced trust in decision support consequently influenced how prescribers sought guidance.

“...[EP order-sentences] might make us not use the BNF [British National Formulary] as much as we should, or speak to a pharmacist as much as we should, because we think we can see it so therefore that must be the dose." (Interview 1, SHO)

Respondents also cited lack of safety features as contributing to errors. There was an overwhelming expectation that EP should prevent duplications, prompt for correct timings of antibiotics, and intercept obvious incorrect doses. Such views were influenced by previous experience or preconceived expectations of EP. Interestingly, two respondents described safety features that were not supported by the system such as drug-drug interactions.

"...[EP] shows much better interactions, if there [are] like two different medications that have interactions, which you might not realise [...] I think that's a very good thing in the system." (Interview 2, Registrar)

Despite the desire for more decision support, certain alerts were perceived as an unhelpful distraction, especially if out-of-sync with workflow or not relevant. In particular, the venous thromboembolism risk assessment alert was perceived to be inconveniently placed and designed for auditing rather than improving prescribing.

Respondents reasoned that such features should be well-designed and represent imminent safety risks. It was also apparent that certain in-built safety features were ineffective, for example when 'tall-man lettering' to help differentiate cephalosporins went unnoticed.

\section{Prescribing roles and responsibilities}

It was widely acknowledged that junior doctors mostly prescribed on instruction from their seniors; it was suggested that EP further emphasises this dynamic. On ward rounds, juniors would be logged into the system to enter notes, and manoeuvre the COW. By default they were therefore responsible for prescribing and seniors perceived them to be more skilled with EP. Errors occurred when instructions from seniors were incomplete and where seniors lacked awareness of EP limitations such as slow-log-in times or the need to manually specify 
antibiotic start-times. Although juniors prescribed, they also expected their seniors to oversee and review medication. A concern was that this now happened less often due to unfamiliarity with EP.

“...you want your consultant to be able to have a quick glance and pick up anything quickly. Whereas I think it's more difficult for them to do it on this system. So they're like relying on us to do the glancing, when I don't feel like I can... Some of the consultants... love [EP system] and can use it really quickly. But lots of them can't." (Interview 11, FY2)

\section{Prescribing attitudes and culture}

It was apparent that EP had influenced individuals' prescribing attitudes and behaviours. Most expressed a positive view of EP in relation to safety, while acknowledging its limitations. Additionally, some suggested improved attitudes because of the robust audit trail.

“...people's attitudes are a bit better as well, because, you know, you've got your name there, like your whole name there in black and white [...] So it probably makes people more accountable ...." (Interview 10, FY1)

Conversely, three participants had an overall negative view and reduced confidence in EP.

"With the more complex medicines that occasionally get used here, I do find myself double and triple-checking more than I would with the paper charts." (Interview 22, SHO)

Views around prescribing being of low importance were frequently expressed. Participants described prescribing having little priority in medical education, influenced by prescribers increasingly relying on protocols and now decision support embedded within EP. Where a complex patient or a prescriber's lack of knowledge contributed to an error, participants strongly expressed a need for more intelligent decision support. Conversely, others feared advanced technology would de-skill prescribers and advocated improving education and feedback; most prescribers were unaware of their error prior to being invited to participate in the study.

\section{DISCUSSION}

\section{Key findings}

Prescribing errors in the context of EP largely stemmed from three groups of latent conditions: functionality and design of the EP system, organisational decisions around implementation, and prescribing behaviours, suggesting that these may be areas for intervention. Use of EP was specifically linked to error-producing conditions at the level of individual, team, task, environment and technology. Active failure types included slips due to incorrect selection from drop-down lists and rule-based mistakes due to over-reliance on default prescribing suggestions or failing to check for duplicated orders. Using order-sentences and order-sets has potentially encouraged rule-driven prescribing practices that may have contributed to the rule-based mistakes observed. 
The application of Reason's accident causation model also revealed how EP has influenced prescribing practices. Shifts in prescribing responsibilities, changes to individuals' prescribing behaviours and learning needs, and the altered physical prescribing environment were described as contributing to error. While EP can improve safety, ${ }^{7-10}$ our findings add to previous literature highlighting that the success of such technology depends on its design and usability, ${ }^{41}$ as well as its integration within the wider system..${ }^{42,43}$ While some findings resonate with existing literature exploring error causation in paper-based settings, 2,18,26-29 other contributory factors were unique to EP, suggesting some important differences in error aetiology that must be considered in strategies to improve prescribing safety.

\section{Recommendations to improve prescribing safety}

\section{EP industry}

Although participants identified aspects of improved efficiency compared to paper, ${ }^{34}$ they were apparently reluctant to spend extra time addressing inconveniences, such as interpreting confusing interfaces. Such frustrations may introduce a distraction leading to attentional failures, ${ }^{44}$ or encourage workarounds, unsafe practices, and negative feelings. ${ }^{32}$ Complex prescribing functions and poorly designed interfaces have also been reported previously. ${ }^{14-16,31,32,45,46}$ There is an urgent need for EP software designers to revolutionise interface design and address usability issues to meet demands of front-line prescribers working in high-pressured environments. Performing certain tasks should be simplified; designers should carefully consider and where possible incorporate the affordances of paperprescribing, ${ }^{47}$ understanding its more nuanced functions. ${ }^{14} \mathrm{~A}$ particular example highlighted in the present study was the tangibility of gaining a quick, overall view of prescribed medications with a paper drug-chart; a function not afforded by the electronic equivalent.

\section{Local informatics teams}

Local teams should consider providing local solutions to usability problems. Alert fatigue can be reduced by minimising non-essential and untimely alerts. Nevertheless, users expect a certain level of sophistication from EP to enhance safety, namely the prevention of obvious errors and the ability to use common drug nomenclature. Additionally, shorter lists have been advocated to reduce slips due to incorrect selection from drop-down menus. ${ }^{31,48}$

\section{Hospital organisations}

Hospital organisations must ensure their infrastructure supports prescribing at the bedside and discourage prescribing away from the patient. ${ }^{49}$ Availability of multiple COWs during ward rounds may encourage better workload distribution among team members. Organisations should aim to minimise periods of hybrid paper and electronic systems during implementation; the suitability of EP for specialist areas is crucial to achieve this. 
The finding that doctors may review medications less frequently on EP compared to paperprescribing has previously been reported. ${ }^{32}$ Interviewees also reported senior doctors avoiding medication review altogether because they lacked confidence with EP. Organisations should clarify expectations regarding medication review; a balance between improving efficiency and discouraging risky prescribing behaviours is required.

\section{Education and training strategies}

There has been a drive to increase prescribing education in recent years. 2,26,50,51 Future educational strategies should specifically consider how to best prepare prescribers to use EP. ${ }^{52}$ The present study also suggests the importance of raising awareness of EP's limitations and the risks of over-reliance, and addressing new learning needs precipitated by EP such as selecting syringe driver diluents.

\section{Strengths and limitations}

The main strength was that our qualitative approach allowed elucidation of prescribers' perspectives of specific errors. The sampling strategy was robust in that errors were identified by pharmacists, encouraging a more objective discussion compared to exploring self-reported errors or general perceptions alone.2,31 This approach allowed exploration of varying error types, including errors that prescribers were previously unaware of. ${ }^{53}$ The explicit application of a theoretical model allowed for elucidation of underlying latent conditions to target for intervention, such as responsibilities, behaviours, attitudes and culture. Additionally, similarities between our findings and other research using different methods ${ }^{31,54}$ support cumulative validity of the findings.

Limitations are that we included perceptions of a sample of prescribers within one hospital using one EP system; nevertheless, it is likely that commonality will exist in similar organisations. It is possible that participants provided socially desirable responses, were subject to recall bias, or were limited by their own perceptions and awareness as front-line clinicians. Member checking was not conducted but interviews were transcribed verbatim thus minimising scope for selective interpretation. Although theoretical saturation was reached, the sample of 25 interviews was smaller than in similar studies of paper-based prescribing, ${ }^{18,26}$ and not all error types could be explored. Similarly, non-medical prescribers were underrepresented.

Future work should aim to understand causes of a wider range of error types, in multiple organisations using different EP software. Triangulation of data with a robust quantitative study to determine prevalence of prescribing error types would add further validity to this research area. Issues affecting non-medical prescribers should be further explored due to considerable differences in their training and prescribing roles.

\section{CONCLUSIONS}


Prescribing errors were associated with latent conditions associated with functionality and design of the EP system, organisational decisions around implementation, and prescribing behaviours using EP. Application of a theory-based qualitative approach allowed elucidation of new findings about error aetiology in the context of EP; these included shifts in prescribing responsibilities, changes to individuals' prescribing behaviours and learning needs, and the altered physical prescribing environment.

The EP industry should focus on revolutionising interface design and usability issues, bearing in mind the wider healthcare context in which such software is used. Healthcare organisations should draw upon human factors principles when implementing EP to improve safety. Consideration of local environments, infrastructure and training should be considered to address local issues identified.

\section{REFERENCES}

1. Lewis PJ, Dornan T, Taylor D, et al. Prevalence, incidence and nature of prescribing errors in hospital inpatients: A systematic review. Drug Safety 2009;32(5):379-389.

2. Dornan $\mathrm{T}$, Ashcroft $\mathrm{D}$, Heathfield $\mathrm{H}$, et al. An in depth investigation into causes of prescribing errors by foundation trainees in relation to their medical education. London: EQUIP study, 2011.

3. Franklin B D, Reynolds M, Shebl N, et al. Prescribing errors in hospital inpatients: a three-centre study of their prevalence types and causes. Postgrad Med J $2011 ; 87: 739-45$.

4. Ryan C, Ross S, Davey P, et al. Prevalence and causes of prescribing errors: The PRescribing Outcomes for Trainee Doctors Engaged in Clinical Training (PROTECT) study. PLoS ONE 2014;9.

5. Seden K, Kirkham JJ, Kennedy T, et al. Cross-sectional study of prescribing errors in patients admitted to nine hospitals across North West England. BMJ Open 2013;3.

6. Vincent C, Barber N, Franklin, B D et al. The contribution of pharmacy to making Britain a safer place to take medicines. London: The Royal Pharmaceutical Society of Great Britain: 2009.

7. Bates DW, Leape LL, Cullen DJ, et al. Effect of computerized physician order entry and a team intervention on prevention of serious medication errors. JAMA 1998;280(15):1311-1316.

8. Ammenwerth $E$, Schnell-Inderst $P$, Machan $C$ et al. The effect of electronic prescribing on medication errors and adverse drug events: a systematic review. J Am Med Inform Assoc 2008;15:585-600.

9. Nuckols TK, Smith-Spangler C, Morton SC, et al. The effectiveness of computerized order entry at reducing preventable adverse drug events and medication errors in hospital settings: a systematic review and meta-analysis. Systematic Reviews 2014;3:56.

10. Reckmann MH, Westbrook JI, Koh $\mathrm{Y}$ et al. Does computerized provider order entry reduce prescribing errors for hospital inpatients? A systematic review. J Am Med Inform Assoc 2009;16:613-623.

11. NHS England. Safer hospitals, safer wards: achieving an integrated digital care record. 2013. Available at: http://www.england.nhs.uk/wpcontent/uploads/2013/07/safer-hosp-safer-wards.pdf (accessed April 2016).

12. National Information Board. Personalised health and care 2020: using data and technology to transform outcomes for patients and citizens. A framework for action. 2014. Available at: https://www.gov.uk/government/uploads/system/uploads/attachment data/file/384 650/NIB Report.pdf (accessed April 2016).

13. Westbrook JI, Reckmann M, Li L, et al. Effects of two commercial electronic prescribing systems on prescribing error rates in hospital in-patients: A before and after study. PLoS Medicine 2012;9. 
14. Ash JS, Sittig DF, Poon EG, et al. The extent and importance of unintended consequences related to computerized provider order entry. J Am Med Inform Assoc 2007;14:415-23.

15. Campbell EM, Sittig DF, Ash JS, et al. Types of unintended consequences related to computerized provider order entry. J Am Med Inform Assoc 2006;13:547-56.

16. Koppel R, Metlay JP, Cohen A, et al. Role of computerized physician order entry systems in facilitating medication errors. JAMA 2005;293:1197-203.

17. Lewis P. Safety in medication use: Prescribing and monitoring medication. London. Taylor and Francis Group; 2016.

18. Dean B, Schachter M, Vincent $C$, et al. Causes of prescribing errors in hospital inpatients: A prospective study. Lancet 2002;359:1373-1378.

19. Institute of Medicine. Committee on Quality of Health Care in America. To Err Is Human: Building a Safer Health System. National Academies Press (US), Washington DC. 2000

20. NHS England. Human Factors in Healthcare. A Concordat from the National Quality Board. 2013. Available at https://www.england.nhs.uk/wpcontent/uploads/2013/11/nqb-hum-fact-concord.pdf (accessed June 2017).

21. Carayon P, Schoofs Hundt A, Karsh BT, et al. Work system design for patient safety: the SEIPS model. Quality and Safety in Health Care, 2006;15(suppl_1):i50-i58.

22. Wetterneck TB and Carayon P. Safety in medication use: Systems perspective and design. London. Taylor and Francis Group; 2016.

23. Reason J. Human Error- Models and Management. BMJ British Medical Journal 2000;320:768-770.

24. Taylor-Adams $S$ and Vincent $C$. Systems analysis of clinical incidents: the London protocol. Clinical Risk 2004;10:211-220.

25. Slight SP, Howard R, Ghaleb M, et al. The causes of prescribing errors in English general practices: A qualitative study. British Journal of General Practice, 2013;63:615.

26. Ross S, Ryan C, Duncan EM, et al. Perceived causes of prescribing errors by junior doctors in hospital inpatients: a study from the PROTECT programme. BMJ Qual Saf 2013;22:97-102.

27. Tully MP, Ashcroft DM, Dornan T. et al. The causes of and factors associated with prescribing errors in hospital inpatients: a systematic review. Drug Saf 2009;32:81936.

28. Coombes ID, Stowasser DA, Coombes JA, et al. Why do interns make prescribing errors? A qualitative study. Medical Journal of Australia 2008;188:89-94.

29. Leape LL, Bates DW, Cullen DJ, et al. Systems analysis of adverse drug events. ADE Prevention Study Group. Jama 1995;274:35-43.

30. Trbovich P, Shojania KG. Root-cause analysis: swatting at mosquitoes versus draining the swamp. BMJ Quality \& Safety 2017 bmjqs-2016-006229. https://doi.org/10.1136/bmjqs-2016-0062296

31. Brown CL, Mulcaster HL, Triffitt KL, et al. A systematic review of the types and causes of prescribing errors generated from using computerized provider order entry systems in primary and secondary care. Journal of the American Medical Informatics Association : JAMIA 2016;9:122-8.

32. Cresswell KM, Bates DW, Williams R, et al. Evaluation of medium-term consequences of implementing commercial computerized physician order entry and clinical decision support prescribing systems in two 'early adopter' hospitals. J Am Med Inform Assoc 2014;21:e194-202.

33. Redwood S, Rajakumar A, Hodson J, et al. Does the implementation of an electronic prescribing system create unintended medication errors? A study of the sociotechnical context through the analysis of reported medication incidents. BMC medical informatics and decision making 2011;11:29.

34. Westbrook JI, Li L, Georgiou A, et al. Impact of an electronic medication management system on hospital doctors' and nurses' work: A controlled pre-post, time and motion study. Journal of the American Medical Informatics Association :

JAMIA 2013;20:1150-8.

35. Dean B, Barber N, Schachter M. What is a prescribing error? Qual Health Care 2000;9:232-7. 
36. Pope C, van Royen P, Baker R. Qualitative methods in research on healthcare quality Quality and Safety in Health Care 2002;11:148-152.

37. Ashcroft DM, Lewis PJ, Tully MP, et al. Prevalence, Nature, Severity and Risk Factors for Prescribing Errors in Hospital Inpatients: Prospective Study in 20 UK Hospitals. Drug Safety 2015;38:833-843.

38. Barber N, Cornford T, and Klecun E. Qualitative evaluation of an electronic prescribing and administration system. Quality \& safety in health care 2007;16:271278.

39. Bradley EH, Curry LA, Devers KJ. Qualitative Data Analysis for Health Services Research: Developing Taxonomy, Themes, and Theory. Health services Research. 2007 42(4):1758-1772

40. British Medical Association, 2016. Doctors' titles: explained. Available at file://clwvfandp-001/User02/is012/Personal\%20Profile/Downloads/PLG-doctors-titlesexplained\%20(3).pdf (Accessed November 2016)

41. Wachter R. Making IT work: harnessing the power of health information technology to improve care in England. London, UK: Department of Health. 2016.

42. Cresswell KM, Sheikh A. Undertaking sociotechnical evaluations of health information technologies. Inform Prim Care 2014;21:78-83.

43. Wetterneck TB, Walker JM, Blosky MA, et al. Factors contributing to an increase in duplicate medication order errors after CPOE implementation. Journal of the American Medical Informatics Association : JAMIA 2011;18:774-82.

44. Rivera-Rodriguez AJ, Karsh BT. Interruptions and distractions in healthcare: review and reappraisal. Quality and Safety in Health Care, 2010;19(4):304-312.

45. Ranji SR, Rennke S \& Wachter RM. Computerised provider order entry combined with clinical decision support systems to improve medication safety: a narrative review. BMJ Qual Saf 2014;23:773-780. doi: 10.1136/bmigs-2013-002165

46. Westbrook Jl, Baysari MT, Li L, et al. The safety of electronic prescribing: manifestations, mechanisms, and rates of system-related errors associated with two commercial systems in hospitals. Journal of the American Medical Informatics Association : JAMIA 2013;20:1159-67.

47. Dahl Y, Svanæs D, and Nytrø Ø. Designing pervasive computing for hospitals: Learning from the media affordances of paper-based medication charts. In 2006 Pervasive Health Conference and Workshops, Pervasive Health.

48. Ahmed Z, Garfield S, Jani Y, et al. Impact of electronic prescribing on patient safety in hospitals: Implications for the UK. Clinical Pharmacist 2016;8:153-160

49. Garfield S, Jheeta S, Husson F, et al. The Role of Hospital Inpatients in Supporting Medication Safety: A Qualitative Study. PLoS One 2016.

DOI:10.1371/journal.pone.0153721

50. Nazar H, Nazar M, Rothwell C, et al. Teaching safe prescribing to medical students: perspectives in the UK. Advances in medical education and practice 2015;6:279-95.

51. The UK Foundation Programme Curriculum. The Foundation Programme Curriculum 2016. Available at http://www.foundationprogramme.nhs.uk/pages/curriculumeportfolio/curriculum-assessment (accessed June 2017)

52. C Brown, Reygate K, Slee A, et al. A review of the literature on the approaches used to train qualified prescribers to use electronic prescribing systems. International Journal of Pharmacy Practice. 2016.

53. Reynolds M, Jheeta S, Benn J, et al. Improving feedback on junior doctors' prescribing errors: mixed-methods evaluation of a quality improvement project. BMJ quality \& safety. 2016;0:1-9

54. Mozaffar $\mathrm{H}$, Cresswell KM, Williams $\mathrm{R}$, et al. Exploring the roots of unintended safety threats associated with the introduction of hospital ePrescribing systems and candidate avoidance and/or mitigation strategies: a qualitative study. BMJ Qual Saf 2017;0:1012

\section{Acknowledgements}

We would like to acknowledge $X X$ and $Y Y$ for their contribution to development of the interview schedule. 